\title{
APURDUTM
}

Euskal ikerketen aldizkaria | Revue d'études basques |

Revista de estudios vascos | Basque studies review

$10 \mid 2005$

Numéro $X$

\section{Euskal poesia idazleen artean lehen anderea}

Maria Estibaliz de Sasiola (Deba 1550a - 1611)

\section{Patri Urkizu}

\section{(2) OpenEdition}

1 Journals

Édition électronique

URL : http://journals.openedition.org/lapurdum/84

DOI : 10.4000/lapurdum.84

ISSN : 1965-0655

Éditeur

IKER

Édition imprimée

Date de publication : 31 décembre 2005

Pagination : 287-302

ISBN : 2-86781-409-X

ISSN : $1273-3830$

Référence électronique

Patri Urkizu, « Euskal poesia idazleen artean lehen anderea », Lapurdum [Linean], 10 | 2005, Sarean emana----an 01 avril 2008, kontsultatu 21 septembre 2020. URL : http://journals.openedition.org/ lapurdum/84 ; DOI : https://doi.org/10.4000/lapurdum.84 


\section{PATRI URKIZU \\ UNED}

\section{MARIA ESTIBALIZ DE SASIOLA \\ (Deba 1550a - 1611)}

\section{EUSKAL POESIA IDAZLEEN ARTEAN LEHEN ANDEREA}

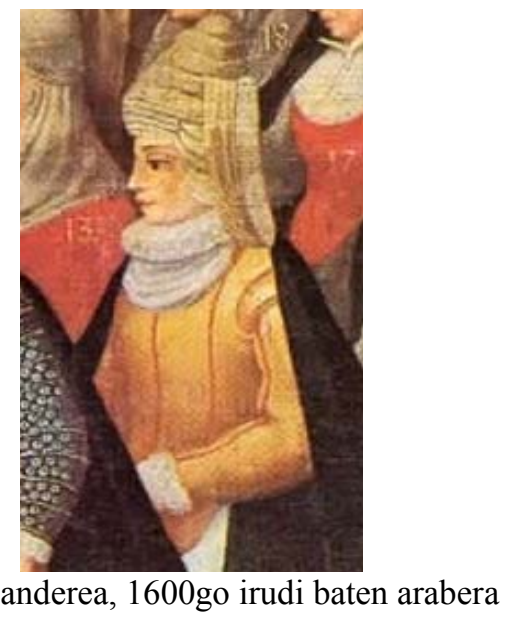

Debako anderea, 1600go irudi baten arabera

Sorthu nintzan Etxauzian,

Hazi Garatian Agerrian, Eskolatü Baiunian,

Madamatü Ürrütian.

Jean de Jaurgain ${ }^{1}$ historialariak dioskunez XIX. menderdian bildu ${ }^{2}$ herri kanta honetan ageri zaigun Etxauzeko anderea, 1422an Menauton Ürrütiarekin ezkondu zen Dominga Ahetzekoa, batzuetan Hauzeko bezala ere agertzen dena litzateke. Eta dirudienez Baxanabarreko jende nobleen alabak Baionan eskolatzen ohi ziren, agian, Santa Klara komentuan, non klarisak kokatu ziren XIII. mendean, eta Saint-Benoit kalean bizitzen ageri diren jada 1309. urtean ${ }^{3}$.

Lehen pertsonan kantatzen duen andereak kexu senarrak ematen dion tratu txarraz, ez zen nornahi, noski, Etxauzeko bizkundearen arreba baizik, eta bazekikeen izkiribatzen. Tamalez, ez zaigu iritsi berak idatzitako eskuzkriburik ez eta Deba aldekoak ziren Milia Lasturko edota Santxa Hortizenik, hauek moldatu eresiak Garibairen bidez iritsi baitzaizkigu.

\footnotetext{
${ }^{1}$ Jean de Jaurgain, "Quelques légendes poétiques du Pays de Soule”, La Tradition du Pays Basque, Paris, 1899, 368-383.

${ }^{2}$ Patri Urkizu (ed.), Chahoren Kantutegia. Susa, Zarautz, 2006.

${ }^{3}$ Pierre Hourmet, Histoire de Bayonne des origines à la révolution française de 1789. Bulletin de la société de Lettres et Arts de Bayonne, Bayonne 1986, 104.
} 
Dena den badakigu garaiko andere noble eta monja euskaldunek bazekitela idazten hala nola Isabel Lobiano Mutrikuko Santa Katalina komentutik ihes egiten lagundu zuen monja ${ }^{4}$, edota Urtubiako anderea XVI. mende bukaeran espioitza lanetan aritu izan zena ${ }^{5}$.

Areago, Lazarragaren eskuzkribuaren aurkikuntza harrigarriak beste datu benetan interesgarri baten aurrean ezartzen gaitu, hots, besterik agertzen ez den bitartean, euskal poesia idazleen artean lehen anderearen eskuzkribuen aitzinean, hots, Maria Estibaliz Sasiola debarraren hiru poemen aurrean ${ }^{6}$.

Nor zen andere hau?

Lope García de Salazar (1392-1476), Bienandanças y Fortunas liburu ospetsuaren egileak honela deskribatzen zituen Debako ahaide nagusiak:

En la villa de Deva ay dos linages de buenos escuderos e de grandes faziendas que fueron e son de las regicas(?) del solar de Olasso el uno es linage del Prevoste de Deva que son naturales de Iziar e fueron e son prevostes de aquella Villa que esta Iziar sobre esta villa de Deva; otro linaxe ay que se llaman los de Sasiola que otrosí fueron buenos escuderos e mucho fazendados e son contrarios los unos de los otros ${ }^{7}$.

Estibaliz Sasiola, beraz, leinuru oneko zen eta balirudike Lazarragatarrekin, Velascotarrekin eta Garibaitarrekin harremanak izan zituela eta Felipe IIaren gortean ibili izan zela, Euskal Herriko beste familia nobleetako hainbat andere bezala.

Nolakoa zen gortea garai haietan?

Lezon jaio eta Abate Marchenaren seme omen zen Eugenio de Ochoa (18151872), idazle eta erudito liberalak erruz idatzi zuen: poemak, eleberriak hala nola Auto de fe Felipe IIaren garaian kokatua, saioak teatroaz eta Espainako literaturaz, etabar. Hainbeste obratarik Epistolario español, Colección de cartas de españoles ilustres antiguos y modernos argitaratu liburuan, hainitzen gutunen artean (Santillanako markesa, Sanchez de Brozas, Agirre kardinala, Juan Iriarte, Leandro Fernández de Moratín, ...) bada bat Eugenio de Salazar (Madrid 1530-Valladolid 1602) delako poeta batena, urterik gabekoa baina 1567 a baino leheangokotzat jotzen duena, non ondoko hitzok irakur ditzakegun:

Está la córte, allende de esto, llena de gentes extranjeras de diversas naciones; encontraréis por las calles unos que os saluden con : beso la mano de vmd.; otros os dicen: beso as maos a vosa mercé; otros: agur xaona, orduan çagoçala; otros: bon giorno, mi ricommendo a la signoria vostra; otros: musieur, je me recommande à vostre bonne grace; otros: Got berliena huberlib den gudem dag; otros: gutmara gad boe. De manera que hay tanta diversidad de saludos, que parece vinieron como guranición y presidio á impedir el daño que el ejército de médicos cortesanos pretende siempre hacer en la gente. Y hay tanta diversidad de lenguas entre ellos, como entre los que edificaban la torre de Babel .

\footnotetext{
${ }^{4}$ Jose Maria Satrustegi, Euskal testu zaharrak. RALV-Euskaltzaindia, 1987, 31-39.

5 José Manuel Floristán, "Conflictos fronterizos, espionaje y vascuence a finales del siglo XVI: 20 documentos inéditos", Fontes Linguae Vasconum, Iruñea 1993, 177-219.

${ }^{6}$ Patri Urkizu (ed.), Joan Perez de Lazarraga, Dianea \& Koplak. Madrid 1567. Erein, Donostia 2004.

${ }^{7}$ Lope García de Salazar, Bienandanzas y fortunas, Libro 21, f-5v. Ikus Fundación Sancho el Sabio, http: //www.euskadi net.
} 
Beraz, ohar gaitezke euskaldunek ere bazutela espainol, portuges, italiano, frantses, flamendar eta ingelesen artean bere lekutxoa gortean. Eta ez da harritzekoa kontutan hartzen badugu zenbat secretario eta zenbat militar eman zituzten euskal probintziek Espainako erresumari.

XVI. mendera hurbiltzen bagara, eta gutun hori idatzi zen garai beretsutara, badakigu zehazki Madrid aldean izan zela Joan Perez de Lazarraga, euskal eleberrigile eta poeta, zeinen eskuzkribuetarik bat argitaratu ahal izan genuen duela gutxi. Bertan argitaratzen genituen ikerketa, transkripzio modernoa, hizkuntzaz eranskin zenbait, ilustrazioak eta eskuzkribuaren faksimilea, eta gainera esaten genuen bazirela hiru poema esku ezberdinez idatziak, XXXIII, XXXIV eta XXXVa bezala sailkatzen genituenak, zeinen buruan hau irakur zitekeen: De la Señora $M^{a}$ Estibaliz de Sasiola.

Aipatzen genuen ere nola Sasiola Debako etxe noble bat zen ( gaur egun etxe bizitza ezberdinez osatua), nola Lope de Sasiola batek Zumaian 1340. urtean beste bat eraiki zuen, eta nola Zumaiako San Pedro kaperan Sasiolatarren armak ageri ziren: San Antonioren hiru gurutze eta hirusta.

Irrikitan gelditu ginen jakiteko gehiago Estibalitzen bizitzaz eta honen familiaz. Liburu hura argitaratu ondoren eta bi urte pasatu direlarik ikusita ez dela ikerketa zehatz eta dokumentatu berririk egin ona hemen aurkitu dugunaren primizia, jakinik ikerketa serios asko egin beharrezko direla oraindik artxiboetan.

Hor ageri dira Martin Ochoa de Sasiola, Gaztelako kondestablearen sekretarioa izan zena Karlos Va enperadorea errezibitu zuena 1539ko urriaren 25ean San Adriango bortuan, eta Debako San Anton kaperaren fundatzailea, zeina Aranzibiako jauna izan zen eta maiz hauzitan sartua Gipuzkoako Juntetako agirietan agertzen denez. Hor Paskual de Sasiola Mutrikuko eskribaua eta Debako alkatea 1583an. Hor Sebastian de Sasiola Debako alkate 1588an. Hor delako Marina, dama vizcayna que servía a la torre de Sasiola, zeina 1604eko urrilaren 4ean hil baitzen. Eta beste...

Baina, nor zen benetan Maria Estibaliz de Sasiola?

Esan behar dugu, gure ikerketa apurren ondoren, debarra zela eta 1550a aitzin jaioa. Zergatik? Ez dugulako bere jaiotze agiririk aurkitu, baina bai heriotzarena eta beste bat, non bere izena ageri den, eta Santa Maria de la Asunción, Debako elizan bataioen data mugak 1550-1607 direlako.

Hona bada aurkitu agirien faksimileak eta transkripzioak :

\footnotetext{
${ }^{8}$ Eugenio de Ochoa (ed.), Epistolario español. Colección de cartas de españoles ilustres antiguos y modernos. Selección y notas de... Biblioteca de Autores españoles, Tomo sexagésimo segundo, Madrid $1965,283$.
} 


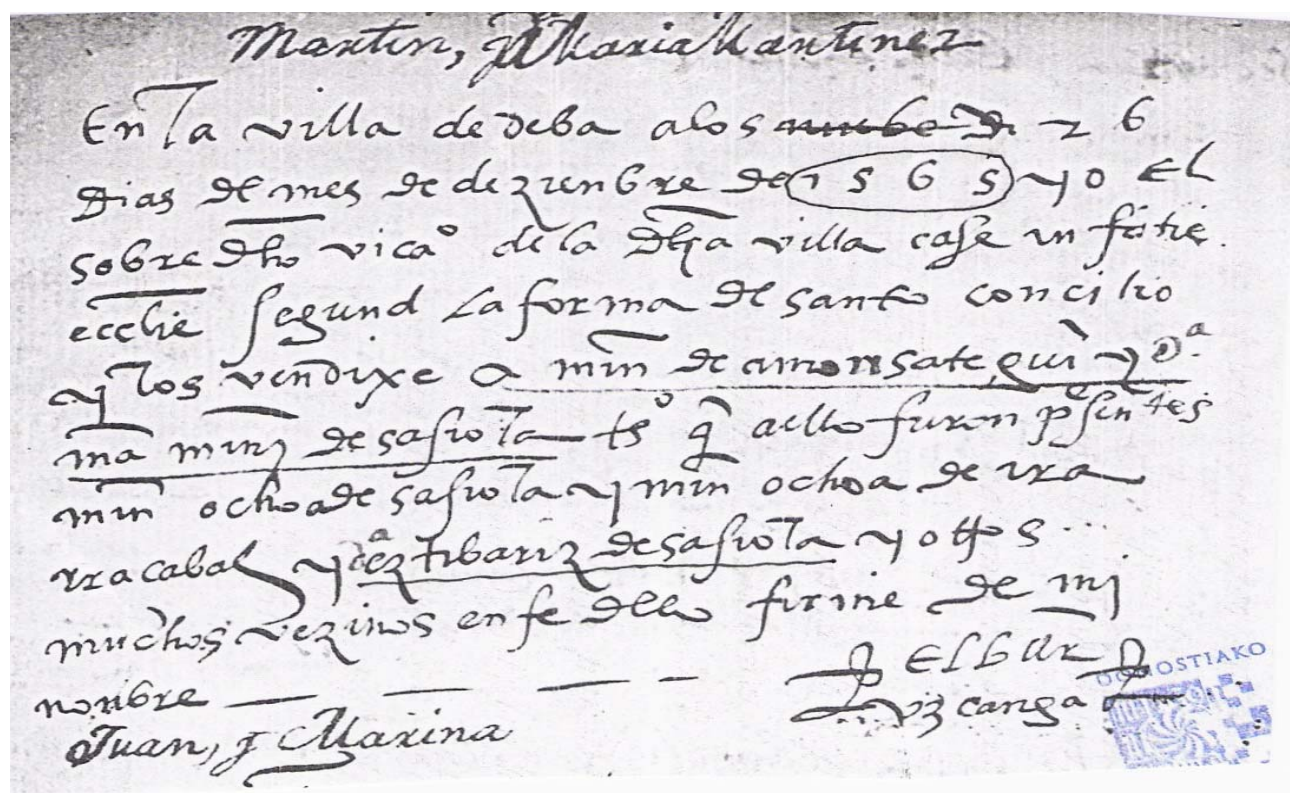

1565Martin y María Martinez

En la villa de Deba a los 26 días de mes de diziembre de 1565 yo el sobredicho vicario de la dicha villa casé en setiembre segund la forma de santo concilio y los vendixe a Martin de Amonsategui y Doña María Martinez de Sasiola testigos de ello fueron presentes Martín Ochoa de Sasiola y Martín Ochoa de Irarrazabal y Doña Estibaliz de Sasiola y otros muchoz vezinos en dello firme de mi nombre Elbar Uzcanga (?). Juan y Marina?.

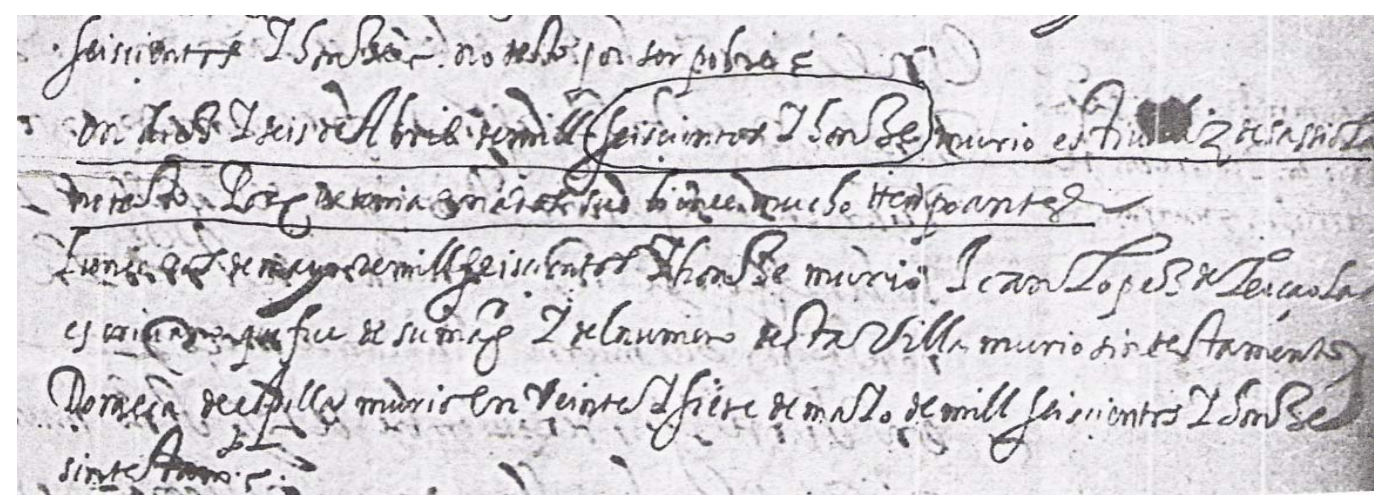

1611

En diez y seis de abril de mill seiscientos y honze murio Estivaliz de Sassiola. No testó porque se tenía donados sus bienes mucho tiempo antes $^{10}$.

Delako Martin Ochoa de Sasiolaz zerbait esan dugu, baina nor ote zen Martín Ochoa de Irarrazabal?

\footnotetext{
${ }^{9}$ Gipuzkoako Eliz barrutiko artxiboa. Sanra Maria de La Asunción-go eliza, f. 2v, 1824 / 001-02. Microfilm 124-02.

${ }^{10}$ Gipuzkoako Eliz barrutiko artxiboa. Sanra Maria de La Asunción-go eliza, f. 263 r. 1824 / 002-05.

Microfilm 124-03.
} 
1600. urtekoa bezala dataturik dagoen agiri ${ }^{11}$ batean ageri zaizkigu Martín eta Domingo Ochoa de Irarrazabal anaiak galeoi kapitanak bezala. Hona nola Edouard Ducéré historialari baionesak aurkezten digun Martin Frantzia eta Espainiako itsasoko gerrez diharduelarik:

Martín Ochoa de Irazabal, capitaine et armateur de la ville de Deva, ajoute que avec les vaisseaux qu'il avait armés à ses frais pendant cette guerre, il avait capturé soixante-dix navires ennemis chargés avec pavillons, étendards et artillerie... n'ayant jamais en avec lui que le capitain Dabile de Aguire, de Deba. (Archives de Bayonne, CC, 164) ${ }^{12}$.

Beraz, eta agiri berri gehiago agertzen ez zaigun bitartean (Estibalizen testamendua, adibidez) aipatu biografiatxoa egin dezakegu. Hots, 1550a baino lehenago jaio zela Deban eta 1611an hil zela, gaztetan hiru poema bederen euskaraz idatzi zituelarik.

Ikus eta azter ditzagun orain hauexek.

Hona hiru poema eskuzkribatu hauen transkripzio modernoa, hots, ez letraz letra, baizik eta gaur egungo Euskaltzaindiaren arauen arabera moldatua, Toponimoak berean utzirik, Gipuzkoa (< Guipuscoa) ezik.

XXXIII

\section{f. $50^{r-1}$ De la Señora $M^{\mathrm{a}}$ Estibaliz de Sasiola}

Leku on bat da Escocia, obeago da Venescia.

Ene aldeko lagun oni elkaenkako flakezia, nai deban orduetako andia dakar fantasia. Bosteun dukat kosta jakan baroe oni balentia, Enperadore jaunaganik ekarritzea lizentzia.

Eleizara juen oi naiz egin oi det bekatu, Ave Maria asi baize ezin oi det notatu. Liburuan bere jarri oi naiz

begi neureok lausotu, fraidea predikatzen jarri, itzik ezin aditu.

Ofrendara Dianea

ondo oi det miratu,

${ }^{11}$ Catálogo de la colección de Vargas Ponce que posee el Museo Naval. (Serie Primera: Numeración Romana). Vol. 1, Madrid, Instituto Histórico de Marina, 1979, 48.

${ }^{12}$ E. Ducéré, Les corsaires sous l'ancien régime. Bayonne, E. Hourquet, 1895, 20. 
sagara gora dianean

amore lindeaz oroitu.

Amore lindo galant orrek //

f.50 ${ }^{\text {r-2 }}$ gogoan, oi ni banindu!

Lagunok baietz diostade,

neuronek ezin sinistu.

Neuronek ezin neio baia

egingo digot mandatu,

arentzat jaio nintzala ta

enagiala largatu.

Ez bisteak kontsolat $<\mathrm{u}>$

ez egonak amorat $<\mathrm{u}>$,

ez majan asentatu,

ez oean albergatu.

Jaun zerukoa eztagizu $<$ la $>$

gizona onein amor $<\mathrm{adu}>$.

Laztan enea, nai gara...

zure atean arteti.

Arren ageri zaitez,

zakuskedan leiotik.

Egun artan nik ez ne $<$ ban $>$

munduan izan bea $<$ rrik $>$,

Esperantzeak bizi oi...

erri onetzat atean.

Esperantzea galdu oi det

Errira natorrenea $<\mathrm{n}>$. //

f. 50 $0^{r-1}$ Ene laztan zuri ederra,

zure laztan ni banari

kontu estua emango digozu

zeruetako jaunari.

Munduan diran podere oro

eskuan dituanari.

Bazinaki gogoa,

laztana biotzekoa,

$<\mathrm{Z}>$ ure minez ilgo banaiz

$<$ l $>$ audatu jaun zerukoa,

$<$ a $>$ more linda galant bata

$<$ e $>$ ne amore berria,

gura dakustan ordu orotan

$<$ or>tzean ur det irria,

$<$ ur $>$ a eztakustanea.

$<$ a $>$ ldea malenkonia

$<$ e>gun ekusi eztet eta

...oi ene bekataria.

... sigarika banendorren

...patu egun batean

... laztanarekin enkontra nezan

$<\mathrm{S}>$ an Juanen aurrean,

$<$ ber $>$ e iru matalotekin

$<$ gorp $>$ utza galanta joean. // 
f. 50 $0^{\mathrm{v}-2}$ Zazpi urtekoz ekarri nau

gaxoturik oea,

zortzierrean irte neban

banijoean kalean,

debotaro entzutera

birjinearen mezea.

Madalena santea da

ene abogadea,

arren, emon degidala

eska nakion doea,

arimakoz eta gorputzekoz

obe dedan bidea.

Ene laztan biotzekoa, kortes, entseinadea,

erri onetan bestek ezteu

ak oi deben librea.

Frantziako armak dakaz

goian eta bean,

aren ekusten etorriko naiz,

Lebante Urrunerean,

biok ondo izango gara

munduan garan artean,

paradisu erreinuan

mundutik goazenean.

Fin.

f. 51 $1^{\mathrm{r}-2}$ Etai lelo ibai lelo, etxean orai leloa, damarika dan galantaena

Belagai Butroekoa.

Belagaia diotsa eta

zorionean jaioa.

Oreanik jagi nintzan

astelen goizean,

eskuetan busti eta

kalera nirteanean

ene laztan zuri ederra

berdurakin aurrean,

soinean gonaz ondo jantzita

jurapa oria gainea,

gerrian gerrikoa eder

solea sotil oinea,

bularteria trapua zuri

labradurak urrea,

dontzellakin fordelisak

lepoan goien ainean.

dontzella batek josi zeban

Iruneko kalean,

niri ondo kosta jakin 
Gipuzkoako lurrean.

Orain aldiaz aita deza $<$ dan $>$

Gaztelako noblezia,

Burgus ta Toledo,

da Cordoba ta Sibillia,

$<$ Marocco erreinua $>$, //

f. 51 $1^{\mathrm{r}-1}$ Barcelona ta Valencia,

Napol erri noblea da,

arík urreant Calabria

Genoako kondaduan.

Pisakin Florencia,

Pullarekin Ferrara baino

obeago da Venescia.

Munduak klaramente an daki

zurea dana Cicilia.

$<\mathrm{A}>$ ostaenez leku ondria,

Toscanarekin Lonbardia,

aek guztiak hamo bere

obeago ene amore onetsia.

Fin.

\section{XXXV}

f. 51 $\quad$ Mendi altuan erurra daidi,

aran baxuan eguzki,

mendiaran guztiak baino

ene koitaok nagusi.

Ikaz lazten ninduan dontzella batek, aurten nai ez ekusi, laster kanbiatu ninduan deseo ebena gaiti.

Ez da erraza, irakurle aditua ohartuko den bezala, konprenitzea erabat hiru testuok, poesiak berez duen zailtasunari erantsi behar baitzaizkio eskuzkribua ez egotea oso-osoa eta hizkuntzaren urruntasuna.

Dena den, esan dezakegu, hiru poemok amodiozkoak direla, XXXIII aren ni poetikoa emakumezkoa dela, XXXIVarena ez dago oso argi, eta XXXVa gizonezkoaren ahotan ipiniak daude bi bertsoak. Garbi dago, halere, hirurak esku berak idatzi dituela, eta kontrako arrazoirik ezean Estibaliz Sasiolak idatziak.

Nola iritsi ziren poema hauek Juan Perez de Lazarragaren poemei atxikitzera? Hona hipotesi bat. Estibalitz, lehen poeman maitemindurik ageri zaigu eta idatziko zion poematxoa, - arrakasta handirik gabe, antza denez, - Juan Ruiz de Velascori. Hau, 
ordea, Belagai Butroekoaz, damarik dan galantaena-z dago maitemindurik. Hirugarrenean, poeta kexu da aurreko urtean maite zuen neskak kasurik egiten ez diolako.

Juan Ruiz de Velascoren semea zen Juan de Velasco Lazarragaren alaba Mariarekin ezkondu zen Santillán de Larrean 1599ko urriaren 31an, eta aitaginarreba hil zitzaionean honen Genealogía... deituari jarraiki zion eta euskarazko eskuzkribuei erantsi Estibalitz de Sasiolarenak bezala jo zituenak.

Irakur dezagun, hain zuzen, delako leinuruei buruzko idaztian dioskuna Juan Ruiz de Velascoz:

El dicho capitán Juan Ruiz de Velasco, hijo de Pedro Ruiz de Gauna y de doña Mencia de Velasco, salio de Galerreta muy muchacho a la ciudad de Valencia, con sus tíos Juan y Pedro de Velasco que vivían en aquella ciudad muy ricos y honrados, porque tuvieron en su tiempo los cargos más honrosos de aquella República. El don Juan de Velasco, que era hermano mayor, fue... y depositario de la persona del duque de Calabria despues que habiéndole conquistado el reino de Nápoles, que era suyo, le tuvo el rey Felipe II detenido en la ciudad de Valencia, encomendando su persona y servicio al dicho Juan de Velasco. Y habiendo estado con los dichos sus tíos cuatro o seis años, se salió en 1558 al reino de Sicilia, asentándose por soldado en la compañia de Adrián García Aragonés, y fué con él a la jornada de los gelbes y la ganaron por marzo del año 1560, e hicieron un fuerte y estando en él fortificando la ciudad llego la armada del turco y tomó y rompió la armada del Rey; y el dicho Juan Ruiz de Velasco con la gente que se halló en defensa del fuerte, se quedó dentro, donde estuvieron sitiados desde 11 de mayo hasta fin de julio de dicho año q560 (donde por falta de agua murieron de 4000 hombres más de 3900), a los cuales los ganó y llevo el truco cautivos a Trípoli de berbería, donde el dicho Juan Ruiz de Velasco estuvo trece meses esclavo hasta que al fin de ellos se rescató a su costa y vino... donde sanó la herida que tenía en la perna derecha, de un arcabuzazo en el asalto de los gelbes le dieron el año 1565[...] Y el Comendador Mayor don Juan de Zuñiga, a la sazón Virrey de Nápoles, le mandó venir con dos galeras, por general de ellas, a la guerra de Portugal donde sirvió hasta su pacificación y después a las islas de la Tercera, hasta el mes de agosto de 1584, y acabado esto con cédula de S.M. de nuevo le entregaron otras dos galeras que habian venido a España de Nápoles con Diego de Medina, enviadas por el duque de Osuna a la dicha guerra, y haciéndole general de ellas por su cédula real le mandó que embarcase en Cádiz el tercio del maestre de campo Manuel de Vega que estaba alli y le mando llevarle a Nápoles; después fué a Barcelona en busca del príncipe Andrea Doria, a quien entonces le habian elegido por generalísimo de la mar, con el cual se fué desde Barcelona hasta Génova donde le dejó... ${ }^{13}$

\footnotetext{
${ }^{13}$ M. Comas, Juan Lopez de Lazarraga y el monasterio de Vidaurreta. Eusko Ikaskuntza, Barcelona, $1936,147-149$.
} 
Badakigu, besteak beste ${ }^{14}$, Juan Ruiz de Velasco izan zela Felipe IIaren konfidantzazko gizona XVI. mende bukaeran eta honek agindutako karitatezko obren banatzaile izan zela, baina poemak lehenagoko giroa ageri digute nabarmen, hots, Mediterraneoko gerlen garaia, eta ingurune honetan behar ditugu noski, kokatu XXXIV poeman ageri zaizkigun toponimoak (Escocia, Venescia, Gaztela, Burgus, Toledo, Cordoba, Sibillia, Marocco, Barcelona, Valencia, Napol, Calabria, Genoa, Pisa, Florencia, Pulla, Ferrara, Cicilia, Toscana, Lonbardia...)

Testuon hizkuntzari errepaso bat ematen badiogu honako xehetasunak azpimarra ditzakegu. Hasi gaitezke grafiekin, eta ikus bokalei dagokionez, idazleak sudurkarien ikurra, hots, $\overline{\mathrm{a}}$ : jācita, (jantzita, XXXIV-13), <n $>$ gisa transkribitu duguna darabilela. Gauza ezaguna $\mathrm{da}^{15}$ gainera garaian bazirela bokal sudurkariak, errimek agertzen diguten eran: nirteanean, aurrean, gainea, oinea, urrea, ainean, kalean, lurrean... (XXXIV-10-24).

Kontsonanteeak direla eta bereizten ditu argi $<-\mathrm{c}^{\mathrm{i}}->$ eta $<-\mathrm{sc}^{\mathrm{i}}->$, egungo $<\mathrm{z}$, tz> , alegia, txistukari frikari eta afrikatu liratekeenak, hala: Escocia, Venescia (XXXIII, 1-2). Halaber $<-$-r- $>$ grafema darabil askotan <-rr- $>$ dardarkari hanitza adieraztekoan, hots, erura $>$ erurra, ecaricea $>$ ekarritzea, ... bezala transkribitu ditugula.

Morfologiari dagokionez Estibaliz Sasiola debatarrarren (D) aditz laguntzaileak, bai iragangaitzak bai eta iragankorrak badu berezitasunik Juan Perez de Lazarraga larreatarraren (L) erekiko, hala, nax (L) / naiz (D); dot (L) / det (D), ... beste askotan berdina izan arren: da, gara, diozu, jakan, nintzala...

Adizkera trinkoz ere aberats dira poemok: EGIN (enagiala, eztagizula...), EKARRI ( dakar, dakaz...), ETORRI (banendorren, natorrenean..., ), IKUSI (dakustan, eztakustanean, zakuskedan...), -IO- 'esan'(diostade, diozu...), IRTEN (nirteanean...), JAKIN (bazinaki, daki,...), JOAN (banijoean, goazenean, joean, natorrenean, ...).

Izenaren bukaerak -a organikoa duten kasuetan Lazarragan bezala -ea gisa bilakatzen da: Dianea, entseinadea, mezea, santea, solea...

Halaber - mente gaztelaniazko atzizkia darabil Lazarraga legez: klaramente, ... Baina, ageri da ere -do atzizkia: ondo...

Ohiko zenbaki ordinalak adierazteko erabili ohi den -garren atzizkia, ikusten dugu laburtua: zortzigarrenean > zortzierrean (XXXIII-72), non agian bokal sudurkaria zitekeen.

Mendebaldeko -rean ablatiboa ere ageri zaigu: Lebante urrunerean ...

Eta garaiko eta geroagoko mendebaldeko euskaran bezala erakuslea erantsi ohi zaio izenari, guk berezita eman arren: baroeoni, lagunok, ...

Joskerari buruz azpimarratuko genuke helburuzko perpausa $-(r) e n$....-t(z)en moldeez adierazten dela, hala: aren ekusten etorriko naiz (XXXIII-88).

Hiztegiari errepasotxo bat emandakoan ikusiko dugu, toponimoez at, badirela maitasun arloko ugari (amore, amoratu, biotzekoa, galant, kortes, laztan, lindo, onetsia...), erlijiozkoak ez dira falta ( ave maria, eleiza, fraidea, jaun zerukoa, mezea, ofrenda, sagara,...), eta jantzi modazkoak bada zenbait ( bularteria, gerrikoa, gona, jurapa, labradura, librea, solea, dontzella batek josi zeban...)

Bada hitz bat ere noblezia munduko Lazarragak eta Sasiolak modu ezberdinean darabiltena: fordelisa (S), flordelisea (L).

Amodiozko hiru poemok, non agertzen zaizkigun besteen artean honako espresabideak (arentzat jaio nintzala, zure minez ilgo banaiz, laztan biotzekoa...,)

\footnotetext{
${ }^{14}$ Adibidez, Juan de Zuñigaren emaztea, Estefanía de Requesens, Felipe IIa haurraren zaindari izan zela, eta bere ama Palamosko kontesarekin gutun ugari gurutzatzu zituela.

15 "La N, alguna vez se pronuncia escondida en las narizes, como en oracioa, ardaoa, y para denotar aquella $\mathrm{N}$ se deve sobre poner una cifra esta manera a modo de nariz" (Pedro de Madariaga, Honra de Escribanos, Valencia, 1565).
} 
nunbait kokatu beharko bagenitu, gaztelaniaz deitu ohi den poesía galante vizcaina barnean litzateke, zeinen lehen adibideak poemok liratekeen.

Eta kontestu zabalago batean txerta nahiko bagenu, XVI. mendeko Errenazimenduko mugimendu barnean izan ziren emakume idazleen artean. Nieves Barandak $^{16}$ erakusten digun bezala errepasoa emanez garaiko emakume idazle espainolei, izan ziren garai hartan Marineo Siculok puellae doctae deitzen zituen zenbait (Isabel de Villena, Sor Juana de la Cruz, Constanza de Castilla, Francisca de Jesús Borja, María de Santo Domingo, Beatriz Bernal, ...) baina emakume idazleak, monjak eta seglarrak ez ziren hasi benetan irakurle zabal eta errekonozimendu publiko bat bilatzen Teresa Avilakoak 1588an Fray Luis de Leonen aurkezpen eta baimenarekin bere obrak argitaratu zireno, eredu bilakatuz.

Maria Estibaliz Sasiola, beraz, orainarte ezagutzen dugunaren arabera giro pribatuan euskaraz poesia idatzi zuen lehen emakumea dugu, Gaztelako gortean antza ibilia, eta agian honen eta besteen ohoretan moldatu zituen bertsook Lazarraga gazteak:

\author{
Oi, Gaztelako errege jauna, \\ erregiagaz ikasu, \\ zeure kortean dama ederrik \\ nola bapere ez dozun, \\ zegaiti Euskel Errian dira \\ eder guztiok dotadu.
}

\footnotetext{
${ }^{16}$ Nieves Baranda, "Las escritoras españolas en el siglo XVI: la ausencia de una tradición literaria propia", Las mujeres escitoras en la historia de la Literatura Española. Lucia Montejo \& Nieves baranda (coord.), UNED, Madrid 2002, 33-55.
} 